\title{
Gemeinsam die Zukunft gestalten: Das Projekt Swiss Library Service Platform (SLSP)
}

\section{Journal Article}

Author(s):

Capatt-Kuppelwieser, Iris; Neubauer, Wolfram (D)

Publication date:

2017

Permanent link:

https://doi.org/10.3929/ethz-b-000199681

Rights / license:

In Copyright - Non-Commercial Use Permitted

Originally published in:

ABI Technik 37(3), https://doi.org/10.1515/abitech-2017-0043 


\section{Fachbeitrag}

\section{Iris Capatt und Wolfram Neubauer}

\section{Gemeinsam die Zukunft gestalten: Das Projekt Swiss Library Service Platform (SLSP)}

https://doi.org/10.1515/abitech-2017-0043

Zusammenfassung: Vor etwa drei Jahrzehnten wurde der erste Bibliotheksverbund der Schweiz gegründet. Seither hat sich im Bereich der wissenschaftlichen Bibliotheken eine stark diversifizierte Verbundlandschaft entwickelt, deren Sinn und deren Zukunftsfähigkeit seit einigen Jahren intensiv diskutiert wird. Mit dem im Jahr 2015 initiierten nationalen Bibliotheksprojekt Swiss Library Service Platform (SLSP) haben sich Bibliotheken und Hochschuleinrichtungen des ganzen Landes erstmals gemeinsam zum Ziel gesetzt, der wissenschaftlichen Community ein breites Spektrum an Dienstleistungen zu vermitteln, zu unterstützen und selbst anzubieten. Der vorliegende Beitrag blickt zunächst auf die Ausgangslage des Projekts zurück, beschreibt dann die Randbedingungen und die erreichten Ziele der ersten beiden Projektphasen und gibt anschließend einen Ausblick auf die Realisierung, die für die Jahre 2018-2020 vorgesehen ist.

Schlüsselwörter: Integriertes Bibliothekssystem (ILS), Service Plattform, Bibliothekskooperation

\begin{abstract}
About three decades ago, the first library network within Switzerland has been founded. Since then a much diversified network landscape has been developed within the domain of scientific libraries. Within the last years the relevance and the sustainability of this situation has been intensively discussed. In 2015 libraries and other academic institutions from all over the country initiated the national library project Swiss Library Service Platform (SLSP), which intends to communicate, to support and to offer a broad package of information services to the science community. The present article looks back to the starting position of the project, followed by a description of the basic conditions and the achieved goals of the first two project phases. Finally there is an outlook to the realization, which is intended for the years 2018-2020.
\end{abstract}

Keywords: Integrated Library System (ILS), Service Platform, Library Co-operation

\section{Einführung}

Vergleichbar zu den Randbedingungen in anderen Ländern, stellt sich auch die Situation der wissenschaftlichen Bibliotheken in der Schweiz als ein einigermaßen komplexes Gebilde dar. Obwohl das Hochschulwesen des Landes mehr oder weniger ausschließlich aus öffentlichen Einrichtungen besteht, bedeutet dies nicht, dass sich hier ein einheitliches Bild hinsichtlich Trägerschaft und/oder Finanzierungs- und Governmentstrukturen ergeben würde.

Insofern unterscheidet sich die Situation in der Schweiz nicht wesentlich von der in anderen Ländern. Ein grundsätzlicher Unterschied zu den Nachbarländern ergibt sich jedoch aus der Kleinteiligkeit der politischen Strukturen, aus der relativ geringen Größe des Landes als Ganzes sowie aus der Mehrsprachigkeit mit ihren drei bzw. vier Sprachregionen. Diese wenigen Punkte verdeutlichen somit bereits die komplexen Randbedingungen, unter denen in der Schweiz Hochschulen, wissenschaftliche Forschungsinstitutionen und die entsprechenden Informationseinrichtungen zu agieren haben.

Trotz dieser Ausgangslage hat sich allgemein die Erkenntnis durchgesetzt, dass die Entwicklung und das Wohlergehen eines kleinen Landes mit nur wenigen natürlichen Ressourcen ganz entscheidend von seiner Innovationsfähigkeit abhängen. Dies hat im Lauf der Jahre dazu geführt, dass Bildung und Wissenschaft, Forschung und Entwicklung einen besonderen Stellenwert einnehmen. So liegen beispielsweise die Forschungs- und Entwicklungsaufwendungen der Schweiz mit etwa 3,4\% des Bruttosozialproduktes (im Jahr 2015) mit Ländern wie Israel und Japan an der Weltspitze, wobei etwa zwei Drittel dieser Aufwendungen von der Privatwirtschaft erbracht werden; dies ist ebenfalls sehr bemerkenswert. Zu erwähnen ist an dieser Stelle auch, dass die meisten Forschungsinstitutionen der Schweiz stark international ausgerichtet sind, was ebenfalls entscheidend zu ihrer hohen Innovationskraft beigetragen hat.

Wir stellen also an dieser Stelle fest, dass Forschung und Entwicklung in der Schweiz einerseits stark international ausgerichtet sind und sich dem internationalen Wett- 
bewerb stellen, dass jedoch andererseits die staatlichen Wissenschaftsinstitutionen nach wie vor vom Föderalismus und den damit verbundenen politischen Randbedingungen entscheidend geprägt werden. Diese wiederum sind wesentlich von breiter Diskussion und kooperativen Entscheidungsprozessen bestimmt.

\section{Das Hochschulsystem der Schweiz und seine Bibliotheken}

Die Hochschulen der Schweiz sind, wie bereits erwähnt, mehr oder weniger ausschließlich in öffentlicher Hand. ${ }^{1}$ So existieren gegenwärtig 10 kantonale, teilweise fachlich fokussierte Universitäten, 2 Technische Universitäten als Einrichtungen der Schweizer Eidgenossenschaft (des „Bundes“), 8 kantonale bzw. interkantonale Fachhochschulen sowie 14 Pädagogische Hochschulen. ${ }^{2}$

An dieser Stelle besonders zu erwähnen ist die seit dem Jahr 2015 auf Basis eines Bundesgesetzes eingerichtete neue Rektorenkonferenz (,swissuniversities“) der Schweizer Hochschulen, deren Aufgabe u. a. in der Interessensvertretung auf nationaler und internationaler Ebene besteht. Darüber hinaus nimmt swissuniversities auch subsidiäre Aufgaben im Rahmen von Förderprojekten und -programmen des Bundes wahr.

Forschungsförderung findet in der Schweiz auf unterschiedlichsten Ebenen statt, doch ist der Schweizerische Nationalfonds (SNF) ${ }^{3}$ der bedeutendste Akteur zur Unterstützung von Wissenschaft, Forschung und Lehre. In diesem Kontext ist allerdings die Information wichtig, dass wissenschaftliche Bibliotheken und Informationseinrichtungen durch die Programme des SNF im Regelfall nicht gefördert werden können, da Infrastrukturförderung nicht zum Förderportfolio gehört.

Alle Schweizer Hochschulen betreiben naturgemäß auch entsprechende Informationseinrichtungen, die von One-Person-Libraries bis hin zu großen Universalbibliotheken mit mehreren Hundert Mitarbeitenden reichen. Daneben gibt es eine größere Zahl anderer Bibliothekstypen, wie (wissenschaftliche) Kantonsbibliotheken, sog.

1 Detailangaben zur Situation der Hochschulen der Schweiz finden sich bei Benitz, S., W. Neubauer. „Die Hochschulbibliotheken in der Schweiz.“ In Bibliothek, Forschung und Praxis 33,3 (2009): 315-327, hier 317-318. Die dort skizzierten Angaben sind mit geringen Änderungen nach wie vor relevant.

2 Detailangaben zum übergeordneten Koordinationsorgan „swissuniversities“ sowie zu den dort vertretenen Hochschulen finden sich unter: https://www.swissuniversities.ch.

3 Vgl. hierzu: https://www.snf.ch.
Studien- und Bildungsbibliotheken, unterschiedlichste Typen von Spezialbibliotheken, Behördenbibliotheken usw. Die Hochschulbibliotheken versorgen die jeweilige Trägerinstitution bzw. deren Mitglieder mit relevanten Informationen, haben jedoch in der Schweiz nicht selten noch weitere Funktionen zu erfüllen. ${ }^{4}$ Die von der Bibliothek der ETH Zürich ${ }^{5}$ ausgeübte Doppelfunktion geht noch einmal in eine etwas andere Richtung, da sie einmal als Universitätsbibliothek fungiert und gleichzeitig als nationales Zentrum für naturwissenschaftlich-technische Information als faktisch landesweite Einrichtung wirkt. Die Bibliotheken der Fachhochschulen bzw. Pädagogischen Hochschulen sind, wie die Trägereinrichtungen selbst, sehr heterogen. Hier gibt es größere, gut organisierte Informationseinrichtungen mit umfangreichen und wissenschaftlich relevanten Beständen, ebenso wie kleine und kleinste Bibliotheken, die nicht immer die kritische Masse einer professionell strukturierten wissenschaftlichen Bibliothek erreichen.

Die im Jahr 1895 gegründete Schweizerische Nationalbibliothek ${ }^{6}$ ist als die nationale Gedächtnisinstitution für das Sammeln, das Aufbewahren und die Bereitstellung aller Publikationen mit Bezug zur Schweiz zuständig und hat ihren Sitz in Bern.

Neben den eigentlichen wissenschaftlichen Bibliotheken existieren eine Reihe von Koordinationsorganen und Serviceinstitutionen für diese Einrichtungen, die allerdings keiner zentral organisierten Leitungsstruktur folgen. Es gibt Einrichtungen, die auf Basis formaler interkantonaler Verträge zusammenarbeiten, ${ }^{7}$ es gibt bibliothekarische Vereine, ${ }^{8}$ es gibt national geförderte Projekte, ${ }^{9}$ und es gibt alle Arten von halboffiziellen bzw. informellen, andererseits jedoch auch verbindlichen Kooperationsstrukturen. ${ }^{10}$

4 Als Beispiele sind hier zu erwähnen: Die Bibliothek der Universität Lausanne (Bibliothèque Cantonale et Universitaire, https://www. bcu-lausanne.ch) oder die Zentralbibliothek Zürich (Kantons-, Stadtund Universitätsbibliothek Zürich, https://www.zb.uzh.ch).

5 Vgl. hierzu: https://www.library.ethz.ch.

6 Vgl. hierzu: https://www.nb.admin.ch.

7 Hierzu gehört beispielsweise der Westschweizer Bibliotheksverbund RERO (https://www.rero.ch).

$8 \mathrm{Zu}$ erwähnen ist hier der sog. Informationsverbund Deutschschweiz; eine koordinierte Zusammenarbeit von vier autonomen Bibliotheksverbünden (https://www.informationsverbund.ch).

9 Das vermutlich umfassendste Bibliotheksprojekt der Schweiz bis zur Initiierung von SLSP war das Förderprogramm E-lib.ch, unter dessen Dach eine Reihe von bibliothekarisch bedeutsamen Teilprojekten abgewickelt wurden. Das Projekt ist mittlerweile abgeschlossen (https://www.e-lib.ch).

10 Hierunter lässt sich etwa das Konsortium Schweizer Hochschulbibliotheken subsumieren. Die operativen Aufgaben dieses Projektes 


\section{Die Verbundlandschaft innerhalb der Schweiz}

So diversifiziert die Bibliothekstypen innerhalb der Schweiz sind, so unterschiedlich sind auch die vorhandenen Kooperationsstrukturen. Betrachtet man die heutige Verbundlandschaft der wissenschaftlichen Bibliotheken in der Schweiz, so fällt auf, dass diese keinem erkennbaren Muster folgt: Die jeweiligen Bibliotheksverbünde sind weder eindeutig nach Sprachregion oder nach Bibliothekstypus differenziert. Vielmehr besteht ein Nebeneinander unterschiedlich strukturierter Verbünde, die teilweise ähnliche Dienstleistungsportfolios bieten, andererseits jedoch organisatorisch ganz unterschiedlich aufgebaut sind.

Die Diskussion über den Sinn und die Zukunftsfähigkeit dieser Netzwerk- bzw. Verbundstrukturen ist allerdings vermutlich so alt wie die regionalen Verbünde selbst, im Falle des Bibliotheksverbundes NEBIS also etwa 30 Jahre. Dieser Verbund ist gegenwärtig der größte in der Schweiz und ist organisatorisch in die ETH-Bibliothek in Zürich eingebunden: Das Netzwerk von Bibliotheken und Informationsstellen in der Schweiz (NEBIS) ${ }^{11}$ ist der einzig mehrsprachige Verbund der Schweiz und umfasst gegenwärtig etwa 150 Bibliotheken aus allen Landesteilen.

Der NEBIS-Verbund wiederum ist Teil des übergeordneten Informationsverbundes Deutschschweiz IDS, ${ }^{12}$ der als Verein konstituiert ist. Diesem gehören noch drei weitere universitäre Verbünde aus der deutschsprachigen Schweiz an (Basel-Bern, Luzern und St. Gallen), wobei alle vier Teilverbünde auf lokaler Ebene autonom agieren. Zum Einsatz kommt in allen Fällen das Softwareprodukt Aleph der Firma Ex Libris.

In der französischsprachigen Schweiz haben sich bisher die meisten Bibliotheken dem Réseau Romand RERO (Réseau des bibliothèques de Suisse occidentale) ${ }^{13}$ angeschlossen. In diesem Kontext ist zu beachten, dass dieser Bibliotheksverbund nicht nur wissenschaftliche, sondern auch allgemein öffentliche und Schulbibliotheken umfasst. RERO wird von den Kantonen Genf, Freiburg, Neuenburg, Jura, Wallis und Waadt als Träger der beteiligten Bibliotheken getragen und finanziert. Als Softwareprodukt

werden von der Bibliothek der ETH Zürich durchgeführt, alle anderen Hochschulbibliotheken der Schweiz sind jedoch durch unterschiedliche Gremien und Entscheidungsprozesse eingebunden. Eine rechtsverbindliche Organisationsstruktur im klassischen Sinn existiert nicht (vgl. hierzu: https://www.consortium.ch).

11 https://www.nebis.ch.

12 Vgl. hierzu Anm. 8.

13 https://www.rero.ch. ist Virtua der Firma Innovative Interfaces in Betrieb. Im Jahr 2015 gab allerdings der Kanton Waadt seinen Ausstieg aus dem RERO-Verbund bekannt, und die Bibliothek der Universität Lausanne betreibt seit diesem Jahr für alle Bibliotheken des Kantons Waadt den Verbund Renouvaud. ${ }^{14}$

Eine weitere Einzellösung wird gegenwärtig an der Nationalbibliothek in Bern entwickelt, die sich nach einem entsprechenden Auswahlverfahren ebenfalls für das Produkt Alma der Firma Ex Libris entschieden hat.

Bereits mit dem Aufkommen der mehr oder weniger integrierten Bibliothekssysteme ab Mitte bis Ende der 1990er Jahre entwickelte sich vor allem in der deutschsprachigen Schweiz eine rege Diskussion dahingehend, ob und wie weit die Zusammenarbeit der einzelnen Bibliotheken etwa beim Betrieb eines Bibliothekssystems oder im Bereich Medienkatalogisierung reichen sollte. Die bereits eingangs erwähnten föderalen politischen Strukturen und die unterschiedlichen Interessen der einzelnen Hochschulen bzw. Bibliotheken haben jedoch dazu geführt, dass sich die Verbundlandschaft der Schweiz heute als sehr heterogen darstellt. Abbildung 1 verdeutlicht die gegenwärtige Situation, die dadurch charakterisiert ist, dass neben den beiden großen Verbünden NEBIS und RERO im Bereich der wissenschaftlichen Bibliotheken mindestens noch drei oder vier weitere Verbundstrukturen existieren. ${ }^{15}$ Das Faktum, dass daneben auch unterschiedliche Softwareprodukte zum Einsatz kommen, hat ebenfalls nicht zu einer engeren Kooperation der einzelnen Verbundstrukturen beigetragen.

Trotz dieser, aus gesamtschweizerischer Sicht eher unbefriedigenden Situation, konnte zumindest am Bibliotheksstandort Zürich in den letzten Jahren eine bemerkenswerte Integration zweier Bibliotheksverbünde realisiert werden. Mit dem im Jahr 2013 abgeschlossenen Projekt INUIT ${ }^{16}$ wurden die Bibliotheksverbünde NEBIS und der „Informationsverbund der Universität Zürich“ zum grössten Bibliotheksverbund der Schweiz zusammengeführt. Im Verlauf der Realisierung dieses Projektes konnten einmal wertvolle Erkenntnisse bei der Migration und Dedublierung großer Datenmengen gewonnen werden. Eine ebenfalls wichtige Erfahrung war darüber hinaus, wie die Zusammenarbeit einer größeren Anzahl von

14 Vgl. hierzu: http://www.bcu-lausanne.ch/renouvaud. Zum Einsatz kommt das Bibliothekssystem ALMA der Firma Ex Libris.

15 An dieser Stelle sollte ergänzend darauf hingewiesen werden, dass auch auf der Ebene von Kantons- und Gemeindebibliotheken entsprechende Verbundstrukturen existieren, wodurch das Gesamtbild weiter verkompliziert wird. Vgl. hierzu beispielsweise : https:// www.bvsga.ch/VerbundSG/default.aspx\#Start1 und http://www.bibliotheken-gr.ch/VerbundGR/default.aspx\#Start1.

16 Vgl. hierzu: http://blogs.ethz.ch/inuit/. 


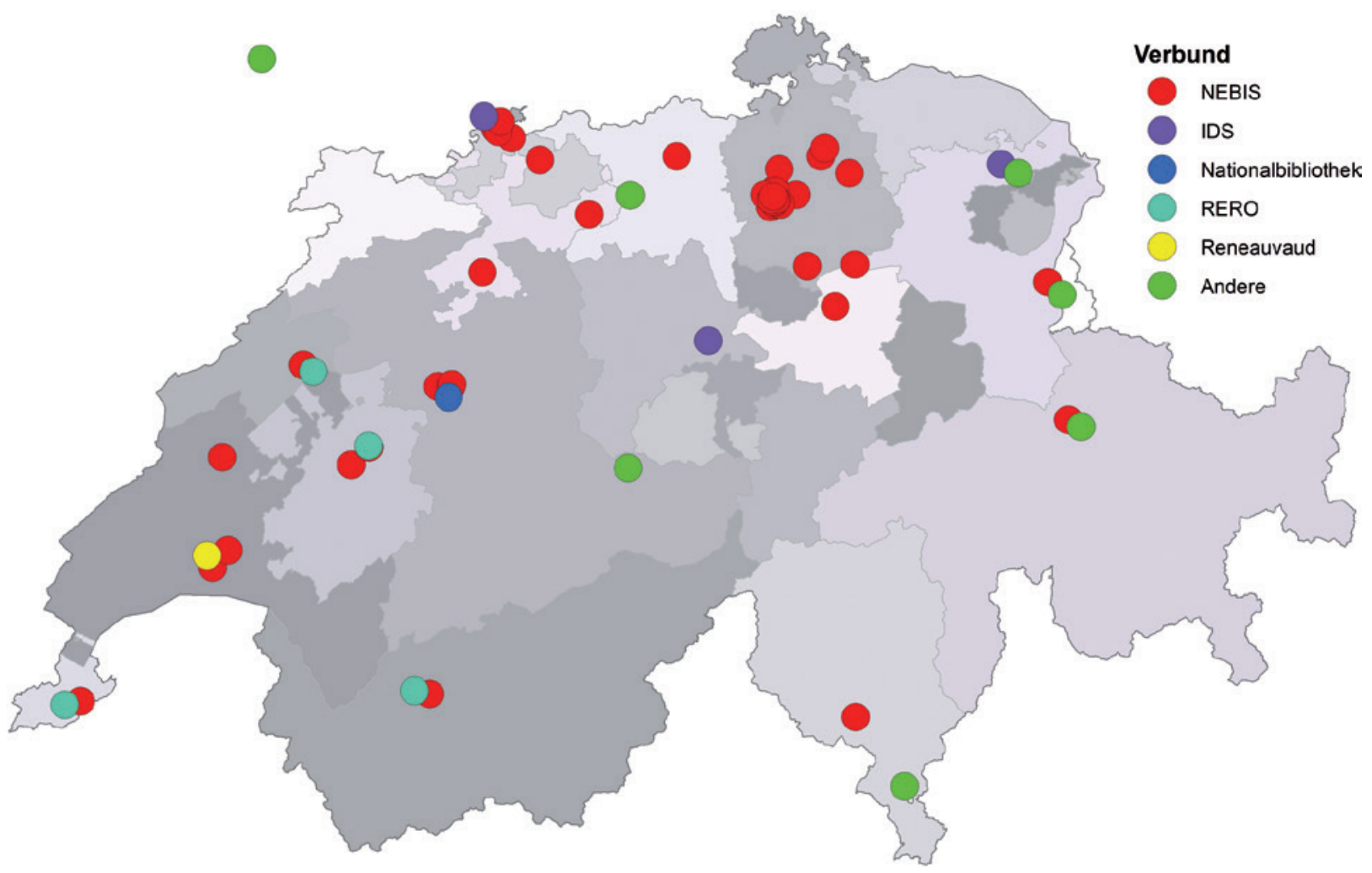

Abb.1: Die gegenwärtige Verbundlandschaft der Schweiz

Mitarbeiterinnen und Mitarbeitern verschiedener Institutionen sinnvoll und zielorientiert gestaltet werden kann.

Ein nicht vorhersehbares Ergebnis des Projektes INUIT war zudem der sich während der Realisierungsphase entwickelnde Ansatz, die dort gemachten Erfahrungen in eine neue, weit größere Projektidee zu überführen. So wurde an dieser Stelle der Gedanke entwickelt, ob es nicht möglich und sinnvoll sein könnte, ein Integrationsprojekt für alle Bibliotheksverbünde der Schweiz zu entwickeln und damit das Konstrukt NEBIS bzw. ein weiterentwickeltes NEBIS auf die nationale Ebene zu übertragen.

\section{Swiss Library Service Platform (SLSP): Die Idee}

Das Projekt SLSP ist das umfassendste Bibliotheksprojekt, das bisher in der Bibliotheksgeschichte der Schweiz initiiert werden konnte. In seinen Grundüberlegungen geht SLSP einmal zurück auf die erfolgreiche Integration des Informationsverbundes der Universität Zürich mit dem Bibliotheksverbund NEBIS, die letztlich den Ausgangspunkt für eine Ausweitung dieses Ansatzes auf weitere (zumin- dest deutschschweizerische) Hochschulbibliotheken bildet. So gab es bereits kurz nach Abschluss des Projektes INUIT erste konkrete Interessensbekundungen von Hochschulbibliotheken hinsichtlich einer Aufnahme in NEBIS. Parallel hierzu erfolgte der erwähnte Austritt der BCU Lausanne aus dem Bibliotheksverbund RERO, was auch dort der Diskussion über die weitere Zukunft dieses Netzwerkes erheblichen Schwung verlieh.

Neben diesen regionalen und/oder nationalen Randbedingungen gibt es naturgemäß auch eine Reihe weiterer, ganz grundsätzlicher Aspekte, die vor allem die zukünftigen Aufgaben und Möglichkeiten wissenschaftlicher Hochschulbibliotheken betreffen. $\mathrm{Zu}$ erwähnen sind hier in erster Linie die rasanten Entwicklungen des Informations- und Wissensmanagements, wo in rascher Folge neue Handlungsfelder entstehen, auf denen Hochschulbibliotheken Innovationangebote machen müssen. ${ }^{17}$ Hierzu sind natürlich entsprechende professionelle Kom-

17 Vgl. hierzu: Oesterheld, Ch. Von dezentralen Bibliotheksverbünden zur nationalen Dienstleistungsplattform. - Vortrag, gehalten auf dem 106. Deutschen Bibliothekartag in Frankfurt a. Main. Frankfurt a. M. 2017, Folie 3. 
petenzen ebenso notwendig, wie die Entwicklung sinnvoller technischer und organisatorischer Lösungen.

Ergänzend zu diesen Punkten müssen darüber hinaus Wege gefunden werden, wie die Bibliotheken in ihren Routineprozessen dauerhaft entlastet werden können, um auf diese Weise diejenigen Personalressourcen freizuspielen, die für die Erarbeitung neuer, zielgruppenspezifischer Serviceangebote unabdingbar sind. Hier rückt dann die Frage in den Vordergrund, wie dieser Transformationsprozess durch den Einsatz von Bibliothekssystemen der neuen Generation unterstützt werden kann.

Für die spezifische Situation in der Schweiz lässt sich also festhalten, dass ab dem Jahr 2013 mehr oder weniger allen Stakeholdern klar wurde, dass jetzt eine günstige Gelegenheit sein könnte, über die heterogene Verbundlandschaft des Landes nachzudenken und eine gemeinsame Anstrengung hinsichtlich einer sehr viel stärkeren Integration $\mathrm{zu}$ unternehmen. Hierzu setzten sich Bibliothekarinnen und Bibliothekare von 10 Institutionen $^{18}$ zusammen und erarbeiteten unter Federführung der ETH-Bibliothek einen Förderantrag zur Einreichung beim nationalen Förderprogramm SUK P-2. ${ }^{19}$ Generelles Ziel des Antrages war der Aufbau einer zentralen Serviceplattform für die ganze Schweiz, allerdings mit klarem Fokus auf die Bereiche Wissenschaft, Forschung und Entwicklung. Bereits von Beginn an war allen Beteiligten klar, dass der Schwerpunkt aller Bemühungen auf den beiden Elementen Kundenorientierung (Wer sind die Kunden und was erwarten sie?) und Dienstleistungsorientierung (Welches Serviceportfolio sollte angeboten werden?) liegen sollte.

Ebenfalls bereits in der Frühphase stimmten alle Stakeholder dahingehend überein, dass der erste Schritt beim konkreten Aufbau einer Serviceplattform, praktisch das Grundelement aller weiteren Aktivitäten, die Einführung eines Bibliothekssystems der neuen Generation sein sollte, wodurch die heterogene Verbundlandschaft einen beträchtlichen Integrationsschub erhalten würde.

18 Hierbei handelte es sich um folgende Institutionen: ETH-Bibliothek, Bibliothèque Cantonale et Universitaire de Fribourg, Hauptbibliothek der Universität Zürich, Haute Ecole de Gestion Genève (HES-SO), Informationsverbund Deutschschweiz e. V., Réseau des bibliothèques de Suisse occidentale (RERO), Universitätsbibliothek Basel, Universitätsbibliothek Bern und Zentralbibliothek Zürich.

19 Das Förderprogramm SUK P-2 (Wissenschaftliche Information: Zugang, Verarbeitung und Speicherung) von swissuniversities (vgl. Anm. 2) sollte einen wesentlichen Beitrag zur Bündelung der Anstrengungen der Hochschulen bei der Bereitstellung und Verarbeitung von wissenschaftlich relevanten Informationen leisten. Durch die Vergabe von Fördermitteln für einschlägige Projektvorschläge und Projektrealisierungen sollte hier ein Aufbruch in eine optimierte Landschaft für wissenschaftliche Information erreicht werden.

\section{Serviceportfolio von SLSP Basic/immediate Services:}

Hierzu gehören Services, die zum Zeitpunkt der Betriebsaufnahme von SLSP implementiert sein müssen. Zu dieser Gruppe gehören all diejenigen Dienstleistungen, die für Bibliotheken der primären Zielgruppe von Beginn an notwendig sind und somit das Basispaket bilden. Die Mehrzahl dieser Services ist mit dem zentralen Bibliotheksverwaltungssystem verbunden.

\section{Optional/immediate Services:}

In diese Gruppe gehören modular angebotene Services, die zum Zeitpunkt der Betriebsaufnahme von SLSP implementiert sein müssen. Diese Dienstleistungen werden nicht von allen Kunden bezogen, sind jedoch für einige von Beginn an notwendig.

\section{Basic/future Services:}

Hier handelt es sich um für die Zukunft wünschenswerte Services, die jedoch für die Aufbauphase von SLSP keine Priorität haben. Diese Dienstleistungen werden zu einem späteren Zeitpunkt ins Basispaket der SLSP aufgenommen.

\section{Optional/future Serivces:}

Bei diesen Dienstleistungen handelt es sich um in der Zukunft denkbare Services, die modular angeboten und nicht von allen Kunden bezogen werden.

\section{Verwaltungsservices:}

Diese Gruppe von Services ist für die Administration und den Betrieb von SLSP notwendig und wird von allen Zielgruppen/Kunden automatisch bezogen.

\section{Die Vision füir SLSP und die Konzeptionsphase}

Im Verlauf der zweiten Hälfte des Jahres 2014 wurden alle Ideen, Vorschläge und Diskussionsbeiträge aus den genannten Einrichtungen zusammengefasst und mündeten schließlich im Februar 2015 in einen Förderantrag bei swissuniversities, der die Vision einer nationalen Dienstleistungsplattform für wissenschaftliche Informationen formulierte und die für die Erreichung dieser Vision notwendigen Prozesse, personellen und finanziellen Ressourcen und technischen Randbedingungen vorstellte.

Der gesamte Projektablauf sollte sich in drei wesentliche Teilabschnitte gliedern (vgl. Abb.2): Konzeptionsphase (August 2015 bis Februar 2017), Aufbauphase (März 


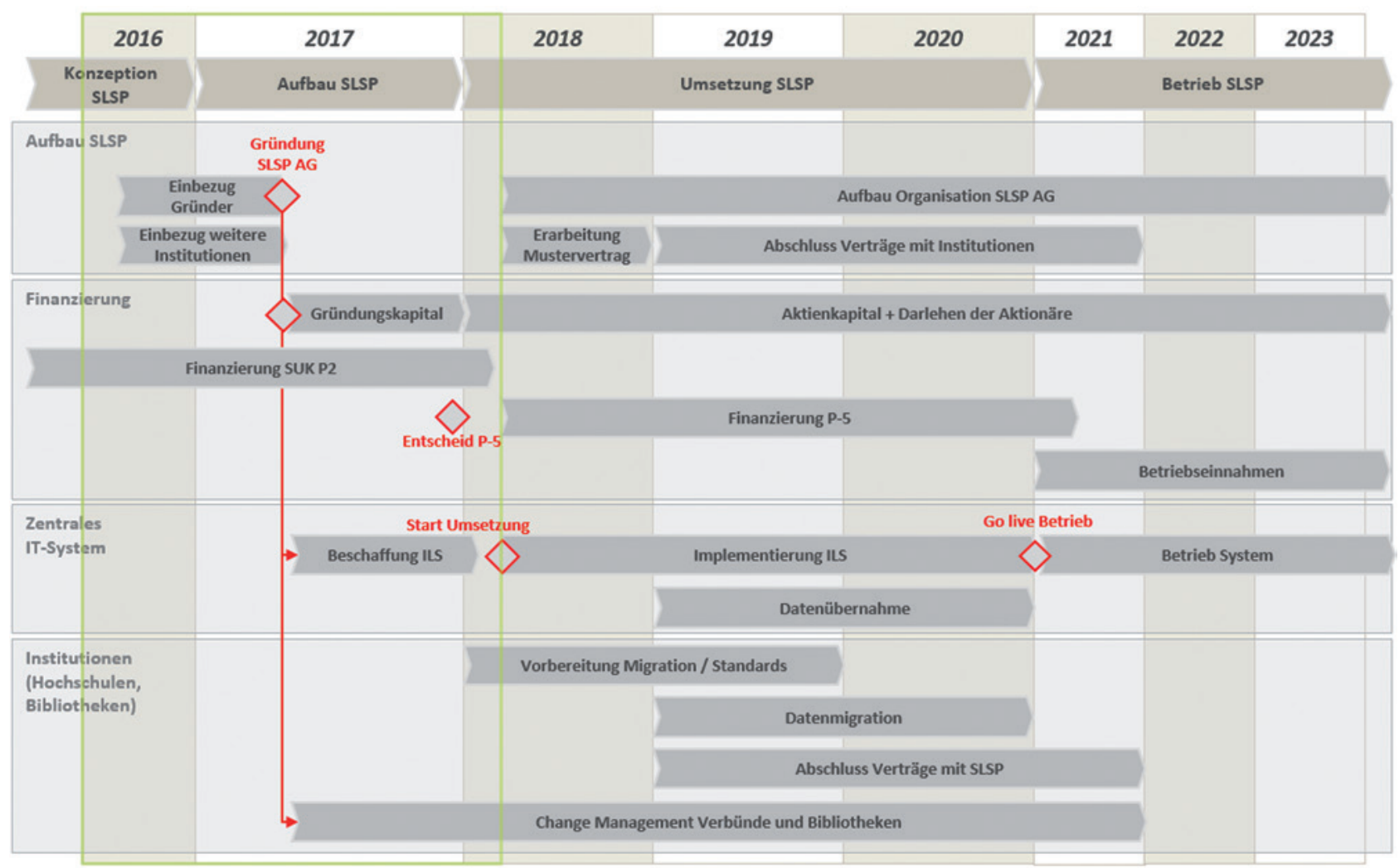

Abb. 2: Roadmap für den Gesamtprojektzeitraum

2017 bis Februar 2018) und Realisierungsphase (März 2018 bis Dezember 2020).

Wie bereits erwähnt, geht die Vision von SLSP als Serviceplattform von einem grundsätzlich zentralen Ansatz aus, was jedoch dezentrale Elemente (wie etwa Lokalredaktionen für Normdaten) nicht grundsätzlich ausschließt. Auch ist beispielsweise denkbar, dass SLSP von dezentraler Seite betriebene Serviceapplikationen (wie etwa bisher dezentral betriebene Datenserver) auf nationaler Ebene „vermarktet“.

Ebenfalls bereits angesprochen wurde im Antrag die grundsätzliche Möglichkeit, durch den Betrieb einer gemeinsamen, einheitlichen IT-Lösung für die gesamte Schweiz Synergien freizusetzen. Arbeiten, die gegenwärtig redundant an vielen Stellen mehr oder weniger gleich geleistet werden, können nun zentral an einer Stelle erledigt werden. Hierdurch besteht zumindest die Möglichkeit, vor Ort die dadurch eingesparten Ressourcen anderweitig einzusetzen und auf lokaler Ebene das Angebot an innovativen und stärker kundenorientierten Dienstleistungen auszubauen.

Naturgemäß war darüber hinaus von Anfang klar, dass sowohl die technische Realisierung, als auch entsprechende Lösungen für Governance und Serviceport- folio das mehrsprachige Umfeld adäquat berücksichtigen müssen, da dies einen entscheidenden Erfolgsfaktor für das gesamte Projekt darstellt.

Die konkreten Ziele der ersten Phase des Projektes SLSP lassen sich folgendermaßen skizzieren:

- Entwicklung eines Serviceportfolios, das sich deutlich an den Bedürfnissen der relevanten Kunden bzw. Kundengruppen orientiert,

- Entwicklung einer sinnvollen Organisationsstruktur für die Plattform, einschließlich einer Konzeption hinsichtlich Governance, Führung und Betrieb,

- Erarbeitung einer technischen Machbarkeitsstudie und Konzeption der informationstechnischen Architektur der zukünftigen IT-Lösung,

- Entwicklung von Vorstellungen hinsichtlich der Finanzierung der Plattform und ihre Darstellung in Form eines Businessplanes,

- Detaillierte Planung der weiteren Phasen des Projektes, Erarbeitung von Plänen zur angemessenen Kommunikation und zum Stakeholdermanagement.

Die konkrete Realisierung des Projektes SLSP orientiert sich prinzipiell an den im Projektführungssystem Her- 


\section{AP 0: Initialisierung}

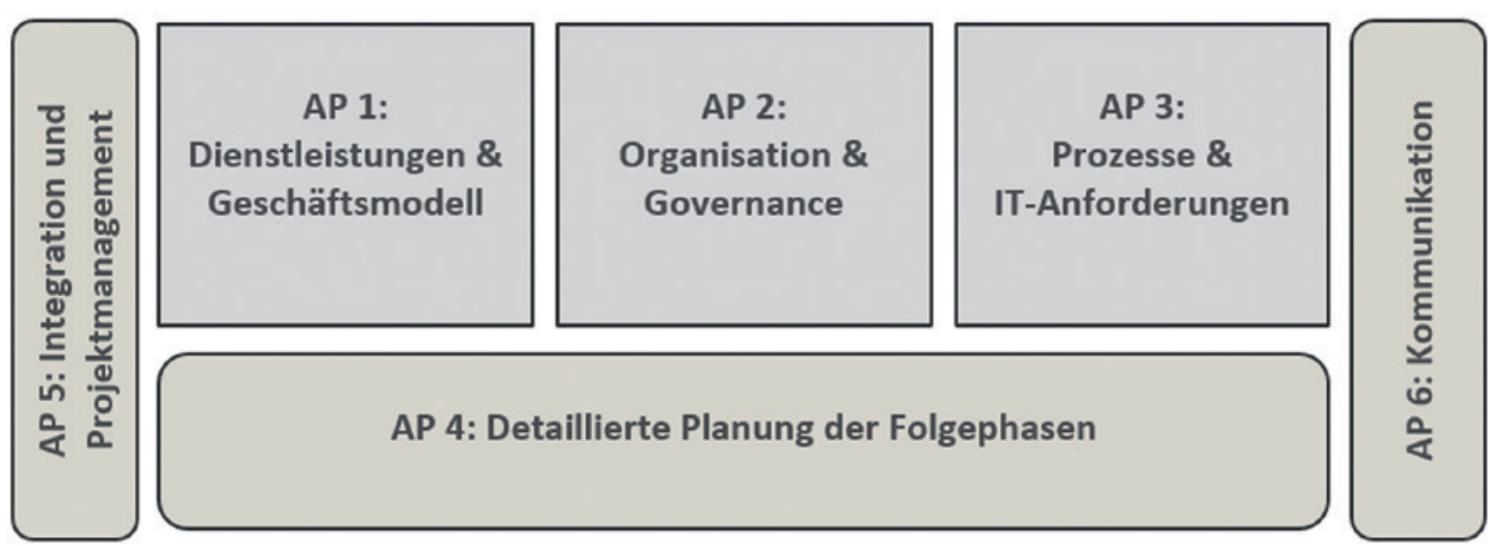

Abb. 3: Übersicht über die relevanten Arbeitspakete der Konzeptionsphase von SLSP

mes $^{20}$ definierten Projektschritten. Hierbei wird das Gesamtprojekt in einzelne Arbeitsschritte, die Arbeitspakete, unterteilt, die dann teils sequentiell, teils parallel abgearbeitet werden. Jedes Arbeitspaket wiederum gliedert sich in einzelne Sub-Arbeitspakete, die sog. Lieferobjekte. In der Konzeptionsphase wurden insgesamt 46 dieser Lieferobjekte schwerpunktmässig in den Arbeitspaketen „Dienstleistungen und Geschäftsmodell“, „Organisation und Governance“ sowie „Prozesse und IT-Anforderungen“ (vgl. hierzu Abb. 3) bearbeitet. ${ }^{21}$

Während der etwa 18 Monate dauernden Konzeptpha$\mathrm{se}^{22}$ wurde eine Vielzahl von einzelnen Ergebnissen erar-

20 Bei Hermes handelt es sich um ein Projektführungsinstrument, das etwa seit 1975 in immer wieder neuen Versionen in der Bundesverwaltung der Schweiz im Einsatz und für IT-Projekte verbindlich ist. Vgl. hierzu: http://www.hermes.admin.ch/onlinepublikation/ index.xhtml.

21 Jede Arbeitsgruppe umfasste etwa 8-12 Mitglieder aus allen Teilen des Landes sowie aus unterschiedlichen Bibliothekstypen. Insgesamt waren etwa 60 Bibliothekarinnen und Bibliothekare an der Konzeptionsphase beteiligt, die zusammengenommen etwa 1700 Personentage einbrachten. Der größte Teil der in den entsendenden Institutionen anfallenden Kosten wurde von diesen übernommen; lediglich ein geringer Anteil wurde den Institutionen aus dem Projekt zurückerstattet. Jeder einzelne Service wurde in einem eigenen Lieferobjekt detailliert beschrieben, was vor allem auch für eine Kostenzuweisung sehr hilfreich sein wird.

22 An dieser Stelle sollte man berücksichtigen, dass sich die einzelnen Projektphasen in der Realität natürlich nicht eindeutig voneinander abgrenzen lassen. Die monatsscharfe Abgrenzung dient primär der Antragstellung. beitet, so dass an dieser Stelle nur die wichtigsten genannt werden sollen: ${ }^{23}$

- Identifikation von Kunden und Kundengruppen,

- Lancierung und Bewertung eine Umfrage bei allen relevanten Stakeholdern hinsichtlich der Serviceanforderungen an SLSP,

- Entwicklung einer Servicematrix,

- Definition von Varianten für eine sinnvolle Governance-Struktur,

- Übersicht über den Markt für relevante Bibliothekssysteme,

- Entwicklung erster Varianten für eine mögliche Systemarchitektur,

- Planung der weiteren Teilphasen des Gesamtprojektes SLSP,

- erste Varianten eines Businessplanes,

- Ausarbeitung und Einreichung eines weiteren Förderantrags (für Phase 2).

$\mathrm{Zu}$ all diesen Themen wurden umfangreiche Abklärungen und Ausarbeiten erstellt und in ihren wesentlichen Aspekten dem zuständigen Entscheidungsgremium von SLSP (in diesem Falle ist dies das sog. Steuerungsgremium; vgl. hierzu Abb.4) zur Diskussion bzw. zur Entscheidung vorgelegt.

Wie bereits erwähnt, standen die beiden Aspekte Kunden- bzw. Dienstleistungsorientierung von Anfang an im Mittelpunkt des Projektes, so dass hier noch eine etwas

23 Die wichtigsten Ergebnisse finden sich unter folgender Adresse: https://blogs.ethz.ch/slsp/a-propos-du-projet/. Der dort genannte Zwischenbericht ist nicht veröffentlicht. 


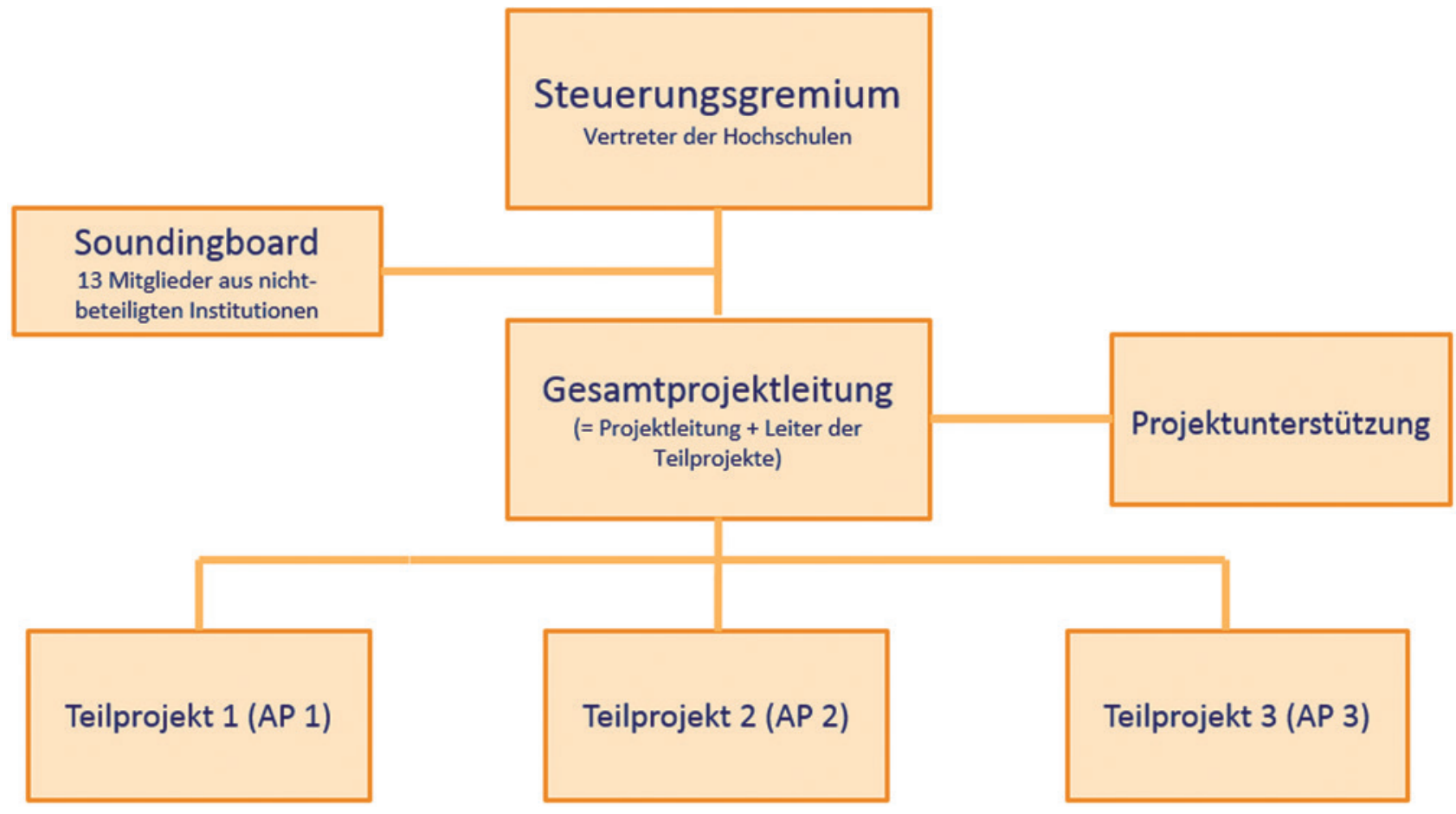

Abb. 4: Organigramm

weitergehende Diskussion angezeigt ist. Alle über die Serviceplattform angebotenen Dienstleistungen wurden letztlich durch eine umfangreiche Bedarfserhebung bei den bibliothekarischen Stakeholdern (vgl. hierzu Abb.5) innerhalb der Schweiz ermittelt. Alle Services sollen grundsätzlich allen Kundengruppen angeboten werden, wobei die optionalen Angebote einen ausreichend großen Kundenkreis abdecken müssen. Alle Dienstleistungen müssen finanziell selbsttragend sein, Quersubventionen sollen möglichst ausgeschlossen sein. Auf diese Weise bleibt die Modularität des Angebotes flexibel, und die Kostentransparenz auf Produktebene bleibt erhalten. Das Gesamtpaket des Serviceportfolios lässt sich so auf die Interessen und finanziellen Möglichkeiten der unterschiedlichen Kundengruppen ausrichten. ${ }^{24}$

Zur Frage, warum der Dienstleistungsaspekt eindeutig im Vordergrund steht, ist an dieser Stelle der Hinweis angebracht, dass SLSP mehr sein wird als ein klassischer Bibliotheksverbund. Es geht in diesem Fall also nicht „nur“ um den Betrieb eines Bibliothekssystems, sondern es geht um eine umfassend angelegte Serviceplattform, die ein breites Spektrum an Dienstleistungen vermittelt (Brokerfunktion), unterstützt (Supportfunktion) und darüber

24 Vgl. hierzu Oesterheld 2017, Folien 6 und 7. hinaus selbst anbietet (Marketingfunktion). ${ }^{25}$ Um überhaupt kundenspezifische Dienstleistungen bestimmen zu können, ist eine Definition der Kundengruppen notwendig, die natürlich nicht zu detailliert ausfallen sollte. Im Fall von SLSP wurden in einem Beteiligungsmodell drei Kundengruppen definiert: Dies sind einmal die „Besitzer“ der Plattform, die in den Aufbau der Plattform investiert haben, über die strategische Ausrichtung entscheiden und selbstverständlich gleichzeitig Servicenehmer, also Kunden sind. Die zweite Gruppe umfasst diejenigen Institutionen, die das sog. Basispaket beziehen (vgl. Abb. 4) und hierfür jährliche Grundbeiträge leisten. Zusätzliche Services werden gesondert bezahlt. Die dritte Gruppe sind Institutionen, die lediglich optionale Services in Anspruch nehmen und hierfür auf Produktbasis bezahlen.

\section{Die Aufbauphase}

Seit März 2017 läuft die zweite Phase des Projektes SLSP, die sog. Aufbauphase, in der wiederum einige für den Projekterfolg wesentliche Arbeitspakete zur Bearbeitung anstehen. Zur Finanzierung dieses Projektschrittes wurde im Kontext der Konzeptionsphase wiederum ein entspre-

25 Vgl. hierzu Oesterheld 2017, Folie 7. 


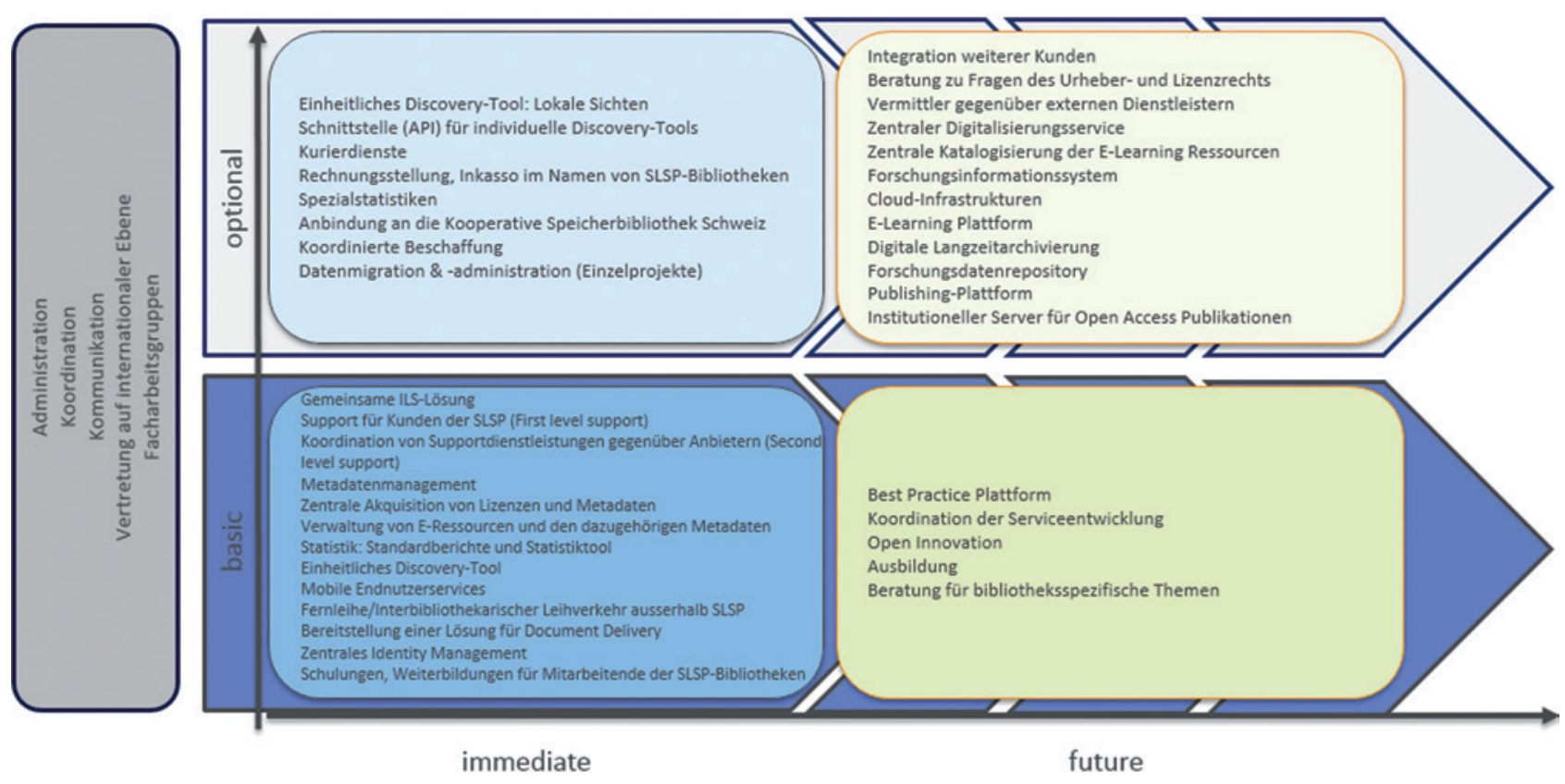

Abb. 5: Servicematrix von SLSP

chender Förderantrag gestellt, der abermals genehmigt wurde und der den Zeitraum bis einschließlich Februar 2018 abdecken wird. Die wesentlichen inhaltlichen Schwerpunkte sind jetzt die Definition einer sinnvollen Governancestruktur, die Ausschreibung für ein Bibliothekssystem der neuen Generation und die Entwicklung eines definitiven Businessplanes, der die realen Kosten für die Realisierungsphase festlegt. Darüber hinaus müssen bereits in diesem Zeitraum wesentliche Vorarbeiten für die Implementierung des neuen Bibliothekssystems und für die Migration der „Altdaten“ aus den laufenden Verbundsystemen in die neue Applikation erledigt werden. Von ganz besonderer Bedeutung ist in diesem Kontext die Frage von gemeinsamen, landesweit geltenden Normen und Standards, da hier in einem mehrsprachigen Land naheliegenderweise eine Vielzahl von Einzelfragen zu klären sind.

Um diese Aufgaben auch problemgerecht lösen zu können, wurde die für die Konzeptionsphase relevante Projektstruktur leicht abgewandelt. Die bisher tätigen Teilprojekte wurden aufgegeben, ${ }^{26}$ und es wurde ein sog. Kernteam ins Leben gerufen, in dem alle beteiligten Institutionen durch ihre Bibliotheksdirektorinnen oder -direktoren vertreten sind. In diesem Gremium finden alle für den Projekterfolg notwendigen bibliothekarischen Diskussionen statt, vor allem auch die Abnahme der Ergebnisse von drei Arbeitsgruppen (Metadaten, Datenmigration und

26 Die dort angesiedelten Arbeitspakete waren weitgehend erfolgreich abgearbeitet.
Benutzung), die für die Aufbauphase ebenfalls neu zusammengestellt wurden. Mitglieder dieser Arbeitsgruppen sind einschlägige Spezialisten aus den beteiligten Institutionen (vgl. hierzu Abb. 6).

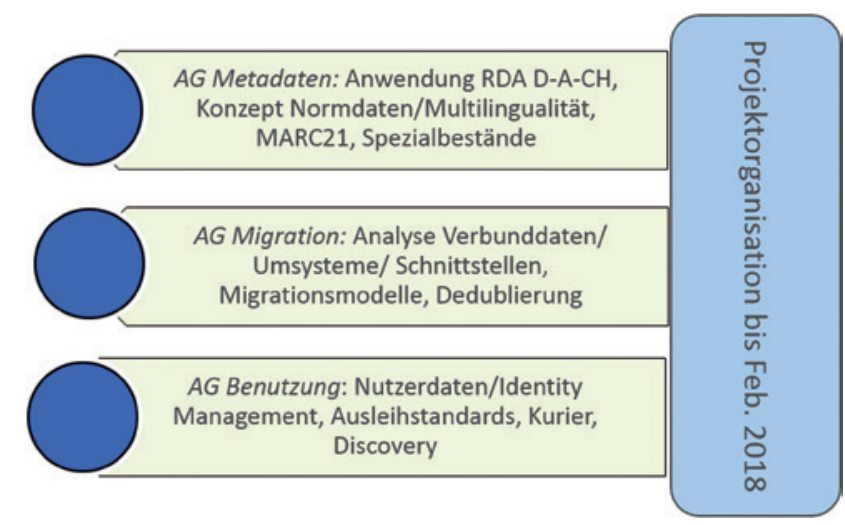

Abb. 6: Bibliothekarische Arbeitsgruppen der Aufbauphase von SLSP

Im laufenden Projektzeitraum (Aufbauphase) wurden bisher folgende für den Projektfortgang wesentliche Entscheidungen getroffen:

- Für die konkrete Realisierungsphase sowie für den Routinebetrieb der Serviceplattform nach dem Jahr 2021 wurde eine SLSP AG gegründet, deren Aktionäre 15 Hochschulen ${ }^{27}$ der Schweiz sind.

27 Berner Fachhochschule, ETH Zürich, Fachhochschule Ostschweiz, Haute Ecole Spécialisée de Suisse occidentale Genève, Uni- 
- Das Gründungskapital der AG beträgt 520000 Schweizer Franken, wobei sich die Gesamtsumme auf elf sog. große (= 40000 Schweizer Franken) und vier sog. kleine (= 20000 Schweizer Franken) Aktienpakete verteilt. Das Führungsgremium der SLSP AG, der Verwaltungsrat, ${ }^{28}$ besteht aus acht Mitgliedern, die von der Versammlung der Aktionäre gewählt werden.

- Der Anforderungskatalog für die Ausschreibung eines neuen Bibliothekssystems wurde durch mehrere bibliothekarische Arbeitsgruppen unter Mithilfe einschlägiger Spezialisten in den ersten Monaten dieses Jahres erarbeitet und in Form einer WTO-Ausschreibung ${ }^{29}$ lanciert. Die konkrete Bewertung und Auswahl wird aller Voraussicht nach im August dieses Jahres erfolgen; die zu erwartenden Vertragsverhandlungen werden dann voraussichtlich im Herbst 2017 stattfinden.

- Die drei genannten Arbeitsgruppen, deren Ergebnisse im Lauf dieses Jahres vorliegen müssen, wurden eingesetzt, damit diese in die Arbeiten der Realisierungsphase einfließen können.

Ebenfalls im laufenden Jahr wird ein Businessplan für die Projektrealisierung in den Jahren 2018-2020 vorliegen, sowie eine konkrete Planung hinsichtlich der ab dem Jahr $2021 \mathrm{zu}$ erwartenden Betriebsphase. Der hierfür notwendige Förderantrag ist ebenfalls ein wichtiges Element der Arbeiten im Jahr 2017. Darüber hinaus ist es notwendig, erste konkrete Schritte für den Aufbau der Geschäftsstelle von SLSP zu unternehmen, die ja möglichst reibungslos in den ersten Monaten des Jahres 2018 in Betrieb gehen soll. Zu erwähnen sind an dieser Stelle Fragen nach dem Gehaltssystem, der rechtlichen Stellung der zukünftigen Mitarbeitenden, Fragen der Altersversorgung, oder die wichtige Frage nach dem Standort der Serviceeinrichtung.

versità della Svizzera italiana, Universitäten Basel, Bern, Freiburg, Genf, St. Gallen und Zürich, Zentralbibliothek Zürich, Zentral- und Hochschulbibliothek Luzern, Zürcher Hochschule der Angewandten Wissenschaften, Zürcher Hochschule der Künste.

28 Es wurde bewusst eine Rechtsform aus der Geschäftswelt gewählt, um auf diese Weise für den Geschäftsbetrieb der Plattform möglichst große Flexibilität zu haben. Der Verwaltungsrat einer AG in der Schweiz ist im Gegensatz zur Situation in Deutschland nicht nur als Aufsichtsorgan tätig, sondern übernimmt auch die Oberleitung der Gesellschaft. Für die Wahl eines Mitgliedes des Verwaltungsrates ist ein Aktienkapital von 80000 Schweizer Franken notwendig. 29 Die Ausschreibung erfolgte unter der Bezeichnung „EvaLIS. Evaluation of a New Library System“ über die entsprechende Plattform für das öffentliche Beschaffungswesen der Schweiz Anfang Juni 2017. Vgl. hierzu: https://www.simap.ch/shabforms/COMMON/ search/searchresult.jsf.

\section{Projektrealisierung und Betrieb der Plattform}

Die konkrete Realisierung des Projektes SLSP ist naturgemäß von zwei wesentlichen Faktoren bestimmt. Dies ist einmal die Bereitstellung der notwendigen finanziellen Ressourcen, die auf zwei Quellen beruhen. Die Planungen gehen einmal von einem erheblichen Beitrag des bereits genannten Förderprogramms P-5 aus und basieren des Weiteren auf einem ebenfalls beträchtlichen Finanzpaket der Aktionäre, also der beteiligten Hochschulen. Über die gesamte Realisierungsphase betrachtet entfallen die notwendigen Aufwendungen im Wesentlichen auf das in Ausschreibung befindliche Softwareprodukt auf der einen und auf die für den Plattformaufbau notwendigen Spezialisten aus dem Bibliotheks- bzw. IT-Bereich auf der anderen Seite. Diese Situation bedeutet, dass zum gegenwärtigen Zeitpunkt hinsichtlich des Gesamtbudgets für die Realisierung der weiteren Planungen noch keine exakten Aussagen möglich sind, also von Annahmen ausgegangen werden muss.

In jedem Fall wird der auf die Aktionäre entfallende Kostenanteil in Form von rückzahlbaren Darlehen geleistet werden, deren Höhe in Abhängigkeit vom jeweiligen Aktienpaket berechnet wird. Die Details dieser Berechnungen werden im Herbst dieses Jahres vorliegen.

Ein in diesem Zusammenhang nicht triviales Problem ist auch die Rekrutierung der für den Plattformaufbau notwendigen Mitarbeiterinnen und Mitarbeiter, die naheliegenderweise weitgehend aus den bestehenden $\mathrm{Bi}$ bliotheksverbünden kommen müssen. Hier ist darüber hinaus zu berücksichtigen, dass die Frage nach der Dauer des Weiterbetriebes der bestehenden Anwendungen, möglicherweise auch des Parallelbetriebes, eine zusätzliche Schwierigkeit für die Übergangsphase darstellt. Entsprechende Lösungsvorschläge werden in der angesprochenen AG Migration erarbeitet.

Über den eigentlichen Betrieb der Plattform SLSP ab dem Jahr 2021 sind zum gegenwärtigen Zeitpunkt naturgemäß nur wenig verbindliche Aussagen möglich. In jedem Fall werden die Betriebskosten für den Routinebetrieb zu 100 Prozent von den Nutzern erbracht werden müssen, da von einer Weiterförderung über ein nationales Förderprogramm nicht auszugehen ist. Hinsichtlich der Höhe der Betriebsaufwendungen gehen die Planungen etwa von der Summe aus, die heute für den Betrieb der unterschiedlichen Verbünde in der Schweiz aufgebracht werden muss. Die Realkosten für die heutigen Verbünde sind allerdings nur schwer zu ermitteln, da man davon ausgehen muss, dass in erheblichem Umfang versteckte Overheadkosten 
anfallen. Somit kann man mit einem gewissen Optimismus davon ausgehen, dass die Kosten für den Betrieb von SLSP niedriger sind, als die kumulierten Kosten heute.

\section{SLSP: Ein Blick in die Zukunft}

Das Bibliotheksprojekt SLSP ist das erste Bibliotheksprojekt der Schweiz, in dessen Konzeption und Realisierung alle Landesteile eingebunden sind. Auch in früheren Jahren gab es landesweit konzipierte Kooperationsaktivitäten, ${ }^{30}$ doch basierten diese Projekte meist auf der Initiative einer einzelnen Bibliothek, bei der weitere Einrichtungen eingeladen waren, mitzumachen. Insofern ist SLSP für die Schweizer Bibliothekslandschaft eine Besonderheit, und es wird sich weisen, ob die einzelnen Institutionen über den gemeinsamen Betrieb eines Bibliothekssystems hinaus ihre Partikularinteressen zurückstellen können und werden. SLSP bietet jedoch erstmals eine landesweite Plattform, um möglicherweise neue, eher forschungsnahe Services (etwa Forschungsinformationssysteme, Data Curation, Forschungsdatenrepositories) über eine zentrale Plattform anzubieten. Daneben bietet eine zentrale bibliothekarische Serviceplattform selbstverständlich die Gelegenheit, auch klassische bibliothekarische Arbeiten ebenfalls zentralisiert zu betreiben. Erwähnt werden sollen hier lediglich die Redaktion und die Pflege national eingesetzter Normen und Standards, die Möglichkeit der zentralen Medienerschließung, oder die Einführung eines nationalen Identity Managements.
Der konkrete Erfolg der Serviceplattform wird ganz wesentlich von den Hochschulbibliotheken der Schweiz selbst abhängen und von deren Bereitschaft, aktiv an der Ausgestaltung des Serviceportfolios mitzuwirken. Der Start des Routinebetriebes zu Beginn des Jahres 2021 ist somit nur der erste Schritt in der Weiterentwicklung des wissenschaftlichen Bibliothekswesens der Schweiz.

\section{Autoreninformationen}

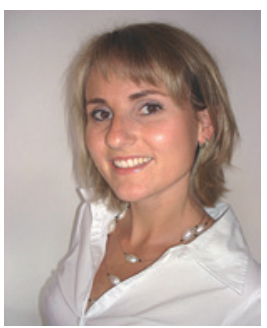

Iris Capatt

Swiss Library Service Platform c/o ETH Zürich, ETH-Bibliothek

Rämistrasse 101

8092 Zürich, Schweiz

iris.capatt@library.ethz.ch

orcid.org/0000-0001-5093-9831

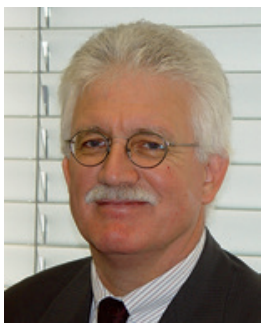

Dr. Wolfram Neubauer

Swiss Library Service Platform c/o ETH Zürich, ETH-Bibliothek Rämistrasse 101 8092 Zürich, Schweiz wolfram.neubauer@library.ethz.ch orcid.org/0000-0002-7999-3054

30 Ein gutes Beispiel für ein solches Projekt ist das Konsortium der Schweizer Hochschulbibliotheken. Vgl. hierzu: http://www.consortium.ch/. 